\title{
Änderungen der KLV, AL und MiGeL per 1. Januar 2019
}

\section{Thomas Kessler}

FMH, Experte, Abteilung Ambulante Versorgung und Tarife

\section{In diesem Artikel werden die wichtigsten Änderungen und Anpassungen der Kran-} kenpflege-Leistungsverordnung KLV, der Analysenliste AL sowie der Mittel- und Gegenständeliste MiGeL per 1. Januar 2019 aufgezeigt.

Per 1.1.2019 ergeben sich Änderungen und Anpassungen der Krankenpflege-Leistungsverordnung KLV, der Analysenliste AL sowie der Mittel- und Gegenständeliste MiGeL. Im nachfolgenden Text sind dabei die wichtigsten Änderungen und Anpassungen zusammengefasst. Weiterführende Informationen sowie Details dazu finden Sie direkt auf der Website des Bundesamtes für Gesundheit BAG.

\section{Änderungen der Krankenpflege- Leistungsverordnung KLV}

Neu wird gemäss Art. 3c KLV die Kostenübernahme bei bestimmten elektiven Eingriffen eingeschränkt («ambulant vor stationär»). Darüber erschien bereits im Juli 2018 eine entsprechende Information in der Schweizerischen Ärztezeitung. Der Artikel 3c KLV tritt per 1.1.2019 in Kraft. Die Details dazu sind im Anhang 1a Art. 3c Ziffer I KLV aufgeführt. Folgende elektive Eingriffe, welche grundsätzlich neu ambulant zu erbringen sind, sind davon betroffen:

1. Krampfaderoperationen der unteren Extremität

2. Eingriffe an Hämorrhoiden

3. Einseitige Hernienoperationen

4. Untersuchungen und Eingriff am Gebärmutterhals oder an der Gebärmutter

5. Kniearthroskopie einschliesslich Eingriffe am Meniskus

6. Eingriffe an Tonsillen und Adenoiden

Bei diesen erwähnten elektiven Eingriffen, die dennoch stationär durchgeführt werden, übernimmt die Versicherung die Kosten für die Durchführung nur, wenn eine ambulante Durchführung wegen besonderer Umstände nicht zweckmässig oder nicht wirtschaftlich ist. Weitere Informationen dazu sind im Anhang 1a Art. 3c Ziffer II KLV, Kriterien zugunsten einer stationären Durchführung, aufgeführt.
Neu wird in Art. 12e Bst. a KLV die Screening-Untersuchung mit "schwerer angeborener Immundefekte» ergänzt.

Die Evaluation der Transkatheter-Aortenklappenimplantation gemäss Punkt 2.2 Herz- und Kreislauferkrankungen, Intensivmedizin wird vom 1.1.2019 bis 30.6.2019 verlängert.

Die Evaluation der Multigenexpressionstests beim Mammakarzinom gemäss Punkt 2.5 Krebsbehandlung wird vom 1.1.2019 bis 31.12.2023 verlängert.

Bei der Schlingenoperation zur Behandlung der Stressinkontinenz bei der Frau gemäss Punkt 3 Gynäkologie, Geburtshilfe gilt neu der Expertenbrief vom 16.6.2016 mit dem Titel «Schlingenoperationen zur Behandlung der weiblichen Stressinkontinenz».

Bei der Positron-Emission-Tomographie (PET/PET/CT) Buchstabe a) Mittels F-2Fluoro-Deoxy-Glucose (FDA) gemäss Punkt 9.2 Andere bildgebende Verfahren unter Indikation 4 gilt: In Evaluation vom 1.1.2019 bis 31.12.2019 bei der Fragestellung «Raumforderung» gemäss den klinischen Richtlinien der SGNM, Kapitel 2.0, vom 28.4.2011 zu FDG-PET.

Die Positron-Emission-Tomographie (PET/PET/CT) Buchstabe f) Mittels PSMA-Tracer gemäss Punkt 9.2 Andere bildgebende Verfahren ist nicht mehr in Evaluation.

Die regionäre Tiefenhyperthermie zwecks Tumortherapie in Kombination mit externer Strahlentherapie oder Brachytherapie gemäss Punkt 9.3 Interventionelle Radiologie und Strahlentherapie wird im Zeitraum vom 1.1.2019 bis 31.12.2020 mit den folgenden Indikationen ergänzt: Tumor-Lokalrezidiv mit Kom- 
Korrespondenz: FMH / Abteilung Ambulante Versorgung und Tarife Baslerstrasse 47 CH-4600 Olten Tel. 0313591230 Fax 0313591238 tarife.ambulant[at]fmh.ch pressionssymptomatik in palliativer Situation, Herdtiefe $>5 \mathrm{~cm}$ und schmerzhafte Knochenmetastasen der Wirbelsäule und des Beckens, Herdtiefe $>5 \mathrm{~cm}$.

Alle Änderungen der KLV im Detail finden Sie auf der Website des Bundesamtes für Gesundheit BAG: https://www.bag.admin.ch/Gesetze \& Bewilligungen/Gesetzgebung/Gesetzgebung Versicherungen/ Gesetzgebung Krankenversicherung/Bundesgesetz über die Krankenversicherung/Änderungen in der Krankenpflege-Leistungsverordnung (KLV).

\section{Änderungen der Analysenliste AL}

Per 1.1.2019 gibt es eine Änderung in der Analysenliste. Für die Position 1368.00 Neugeborenen-Screening auf Phenylketonurie, Galaktosämie, Biotinidasemangel, Adrenogenitales Syndrom, Kongenitale Hypothyreose, Medium-Chain-AcylCoADehydrogenase (MCAD)Mangel, zystische Fibrose, Ahornsirupkrankheit (MSUD), Glutarazidurie Typ 1 (GA-1) und die schweren angeborenen Immundefekte gilt neu: Die Kostenübernahme für die Untersuchung auf schwere angeborene Immundefekte ist befristet bis am 31.12.2024. Angemerkt sei, dass es sich hier nicht um eine Analyse im
Rahmen der Präsenzdiagnostik im Praxislabor handelt, bzw. Ärzte/innen in der Arztpraxis dürfen diese Analyse nicht zu Lasten der Sozialversicherungen erbringen und abrechnen.

Alle Änderungen der Analysenliste im Detail finden Sie auf der Website des Bundesamtes für Gesundheit BAG: https://www.bag.admin.ch/Versicherungen/ Krankenversicherung/Leistungen und Tarife/Analysenliste (AL).

\section{Änderungen der Mittel- und Gegen- ständeliste MiGeL}

Per 1.1.2019 gibt es eine Änderung in der Mittelund Gegenständeliste: Für die Positionsnummer 09.03.01.00.2 Weste mit Defibrillator wird die Evaluation auf den 31.12.2021 verlängert.

Alle Änderungen der Mittel- und Gegenständeliste im Detail finden Sie auf der Website des Bundesamtes für Gesundheit BAG: https://www.bag.admin.ch/ Versicherungen/Krankenversicherung/Leistungen und Tarife / Mittel- und Gegenständeliste (MiGeL). 